\title{
Assessment of bruise volumes in apples using X-ray computed tomography
}

\author{
Elien Diels ${ }^{a, *}$, Mattias van Dael ${ }^{\mathrm{a}}$, Janos Keresztes ${ }^{\mathrm{a}}$, Simon Vanmaercke ${ }^{\mathrm{a}}$, \\ Pieter Verboven ${ }^{\mathrm{a}}$, Bart Nicolai ${ }^{\mathrm{a}, \mathrm{b}}$, Wouter Saeys ${ }^{\mathrm{a}}$, Herman Ramon ${ }^{\mathrm{a}}$, Bart Smeets ${ }^{\mathrm{a}}$ \\ ${ }^{a}$ KU Leuven - MeBioS,Kasteelpark Arenberg 30, 3001 Heverlee, Belgium \\ ${ }^{\mathrm{b}}$ Flanders Center of Postharvest Technology, Willem de Croylaan 42, 3001 Leuven, Belgium
}

\section{A R T I C L E I N F O}

\section{Article history:}

Received 7 October 2016

Received in revised form 26 January 2017

Accepted 26 January 2017

Available online 14 February 2017

\section{Keywords:}

X-ray computed tomography

Bruise

Postharvest

Image processing

Fruit

Non-destructive quality inspection

\section{A B S T R A C T}

A non-destructive methodology was developed to automatically detect and quantify bruise volumes in the equatorial region of apples, using X-ray CT images. Grey level threshold values were calculated to segment bruises in 'Jonagold', 'Joly Red' and 'Kanzi' apple fruit using the multi-level Otsu's threshold method. Comparisons were made between the CT-based bruise volume estimates and bruise volume estimates that were based on destructive measurements in combination with simple geometric assumptions. Visualisation of the bruises in both 2 and 3D showed that bruises, resulting from a pendulum impact with a spherical impactor, can be highly irregularly shaped which implies that the bruise volume estimations based on simple geometric assumptions cannot deliver accurate results.

(C) 2017 Elsevier B.V. All rights reserved.

\section{Introduction}

X-ray computed tomography (CT) is a visualization technique that can render three-dimensional images of the internal structure of agricultural commodities. X-ray CT detects internal structures based on differences in X-ray attenuation resulting from 3D variation in the composition of the material. This makes X-ray CT an excellent technique to visualize the porous structure of products (Cantre et al., 2014; Herremans et al., 2014a, 2014b; Magwaza and Opara, 2014), as X-ray scattering in biological products is limited, while the absorption by air is negligible compared to that by water-rich tissues. As a result, X-ray techniques have been explored to detect several kinds of internal defects in agricultural products. These defects are often detectable with X-ray imaging because they change the tissue density locally or the distribution of the water content (Donis-González et al., 2014; Herremans et al., 2013, 2014a, 2014b; Kotwaliwale et al., 2014; Lammertyn et al., 2003). Bruise damage in apples is one of these defects that has been found to be detectable by using linescanning X-ray equipment (Diener et al., 1970; Schatzki et al., 1997). Besides radiographs of bruised apples from latter research, low quality X-ray CT images of apples showing bruises have been

\footnotetext{
* Corresponding author.

E-mail address: elien.diels@kuleuven.be (E. Diels).
}

published in a study not focused on bruise damage (Tollner et al., 1992).

Apple bruises are caused by failure of cells when the internal pressure exceeds a critical value (Baritelle and Hyde, 2001) and can be identified by the flattening, softening or discoloration of the tissue (Pang et al., 1994). Both softening and browning of the tissue are the result of enzymatic reactions taking place after the enzymes are brought into contact with polyphenols due to rupturing of membranes and cell walls (Li and Thomas, 2014).

Until now, most research into the damage susceptibility of fruit made use of destructive measurements to quantify bruise volume $\left(V_{B}\right)$ (Chen and Yazdani, 1991; Lu and Wang, 2007; Pang et al., 1992; Zarifneshat et al., 2010). Similar to a method based on magnetic resonance (MR) images (Lin et al., 2003), a method using X-ray CT images allows for the investigation of the temporal changes of bruises in fruit, since the measurements are non-destructive. Quantifying bruise damage based on X-ray CT images may solve the difficulty to measure the $V_{B}$ in complex cases including overlapping multiple impacts. A 3D non-destructive CT analysis may also be applied to other agricultural products like tomatoes and strawberries, whose bruises are difficult to quantify by manual measurements, because there is no clear discoloration as in apples (Van Zeebroeck, 2005).

Bollen et al. (1999) propose several formulae for computing $V_{B}$, which all assume that a bruise can be approximated as either an ellipsoid or a section of an ellipsoid or sphere made by a flat or 
convexly shaped cutting plane. A 3D visualization technique may be used to assess the appropriateness of such formulae.

Bollen et al. (1999) and Lin et al. (2003) compared several destructive $V_{B}$ estimation methods with $V_{B}$ estimates derived from RGB and MR images, respectively. The RGB images were made from several parallel slices through the bruises. The brown area in each slice was summed up to calculate the total bruise volume. According to Bollen et al. (1999), the enclosed volume formula with elliptical bruise shape assumption was the most appropriate one for estimating $V_{B}$ only when the bruise widths could be measured across both perpendicular directions and in case both small and large bruises were investigated. In the work of Lin et al. (2003), MR images were made of intact bruised apples. Bruises were automatically segmented from these MR images in a predefined window containing the bruise. The reconstructed bruise volumes were compared to two conventional destructive $V_{B}$ estimation methods, namely the full-depth method and ellipsoid method with circular bruise shape assumption. They concluded that both these methods yield reasonable bruise volume estimates. However, bruise volume estimates using MR were considered more accurate.

In this work, we develop a method to quantify bruise volume $\left(V_{B}\right)$ from X-ray computed tomography (CT) measurements of bruised apples and we compare the presented X-ray CT-based $V_{B}$ estimation method to destructive $V_{B}$ estimation methods. Besides elaborating a new non-destructive technique for bruise size quantification, the high quality X-ray CT images produced in this work permit the observation of bruise shape and features such as cracks, and can help understand spatial changes in water content and tissue density in (and near) the damaged tissue. This is the first study that investigates these characteristics of apple impact bruises of commercially realistic size in 3D.

\section{Materials and methods}

\subsection{Apple samples}

The experiment was carried out on apples (Malus $\times$ domestica Borkh.) of three different cultivars, namely 'Jonagold', 'Joly Red' and 'Kanzi'. These three cultivars are commercial cultivars in Belgium and were chosen for their availability and good storability, and to evaluate bruises in different cultivars. Fifteen apples per cultivar were picked at the end of September 2014 and stored under controlled atmosphere conditions (VCBT, Heverlee, Belgium) for approximately 15 weeks. 'Jonagold' and 'Joly Red' were stored at $1{ }^{\circ} \mathrm{C}, 1 \% \mathrm{O}_{2}$ and $3 \% \mathrm{CO}_{2}$, while 'Kanzi' was stored at $4{ }^{\circ} \mathrm{C}, 2 \% \mathrm{O}_{2}$ and $0.7 \% \mathrm{CO}_{2}$ since these are the optimal storage conditions for long term storage of these cultivars (VCBT, 2014). One day before bruising the apples were taken out of storage, where after they were left at room temperature during the remainder of the entire experiment.

\subsection{Pendulum measurements}

Apple fruit were damaged in a controlled way by releasing the rod of a pendulum device (Fig. 1 ) onto three different places on the equator of the apple that was mounted to the anvil by a rubber band. Five different release angles were used resulting in a wide range of impact energies and consequently bruise volumes. The pendulum device has a spherical aluminum impactor (radius of curvature: $15 \mathrm{~mm}$ ) as previously described by Diels et al. (2016). The encoder (RON 275-TTLx5, Heidenhain, Traunreut, Germany) and force sensor mounted on the device allow for measuring the deformation and force during impact, respectively. Impact energy was calculated as the area under the loading curve.

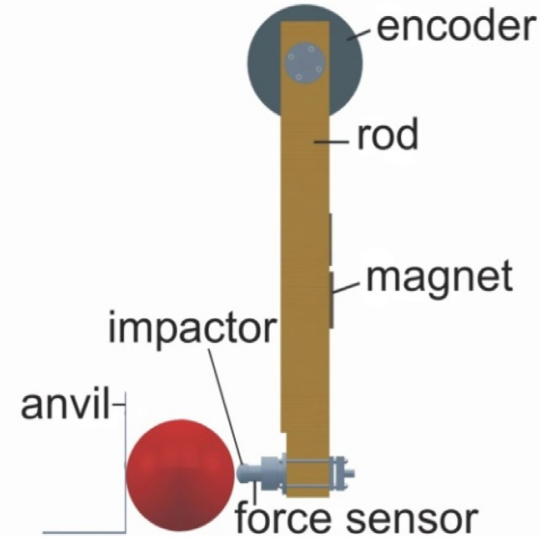

Fig. 1. Schematic representation of the pendulum device.

\subsection{X-ray computed tomography}

\subsubsection{Set-up}

Five days after impacting the 'Jonagold' and 'Joly Red' apples and four days after impacting the 'Kanzi' apples, each apple was scanned with a Microfocus Computer Tomography Tomohawk system (AEA Technology, Harwell, UK). This long time interval between bruising and imaging was chosen to let the bruises stabilise and to allow for plenty moisture removal from the bruises which could enhance the difference in X-ray absorption between bruised and healthy tissue. The X-ray source was operated at a voltage of $80 \mathrm{kV}$ and a current of $439 \mathrm{~mA}$, and the shadow image was acquired by a CCD camera (Adimec MX12P). The apple was placed in a styrofoam box on a rotation stage that rotated over $187.8^{\circ}$ in steps of $0.3^{\circ}$. The total scanning time for one apple was approximately $30 \mathrm{~min}$.

\subsubsection{Image processing}

NRecon (1.6.9.18, Bruker microCT, Kontich, Belgium) was used to reconstruct cross-sectional images from the X-ray transmission images using the filtered back projection method. The reconstruction resulted in 3D stacks of about 900 8-bit greyscale images (image plane perpendicular to the stem-calyx axis, pixel intensity values between 0 and 255) covering the entire apple. During reconstruction, the data were downsampled with a factor of two to reduce the computational load during further image processing in Matlab (R2014a, The MathWorks Inc., Nattick, MA). This downsampling procedure led to an isotropic voxel size of $210.79^{3} \mu \mathrm{m}^{3}$. Further steps to process the CT data are graphically represented in Fig. 2 on a 2D cross-sectional image of a stack. First, a global image threshold was calculated per stack using Otsu's method (Otsu, 1979). This threshold was used to separate the apple from the background (Fig. 2a). Holes in the image stacks were filled and small objects originating from the surrounding styrofoam were removed using an image morphological opening (Fig. 2b). In order to automatically select a region of interest (ROI) (see Fig. 2c) that doesn't includes the core, but does include all the bruises made on the equator of the apple, the centroid location of the segmented apple was used as the centre of the circle (radius $17 \mathrm{~mm}$ ) defining a cylinder that was excluded from the ROI. Thereafter, the multilevel Otsu's method (Otsu, 1979) was used to calculate several thresholds per apple that converted the greyscale image stacks into image stacks with several discrete levels (Fig. 2d), whereby the discrete level corresponding to regions with intensities that were 
(a)

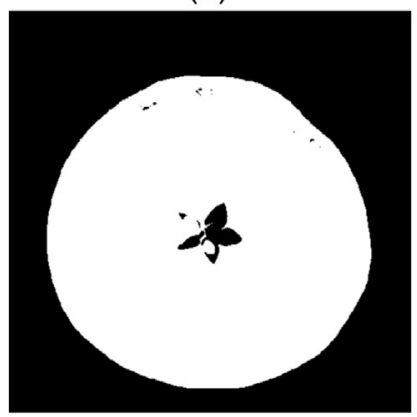

(d)

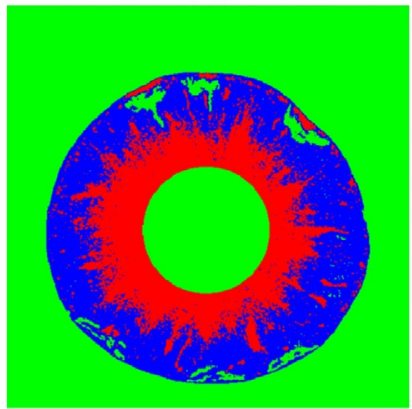

(g)

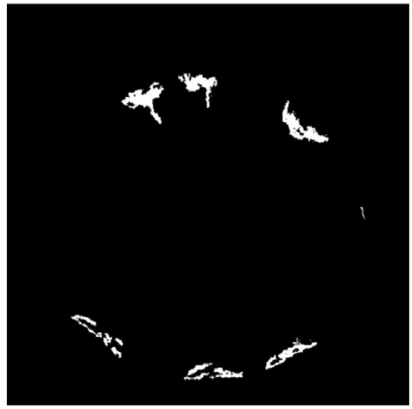

(b)

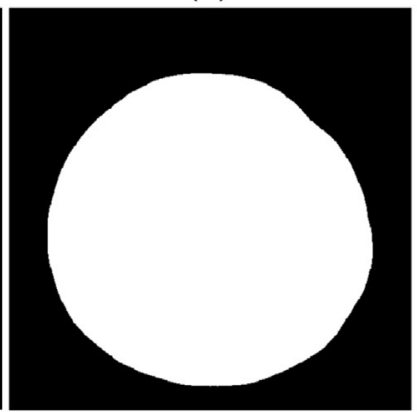

(e)

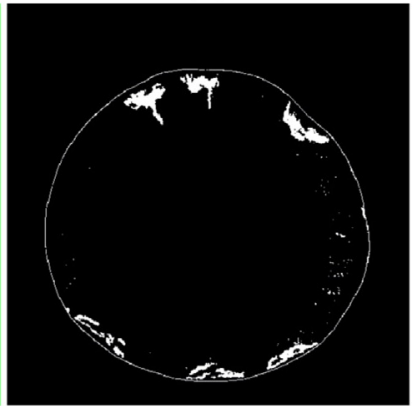

(h)

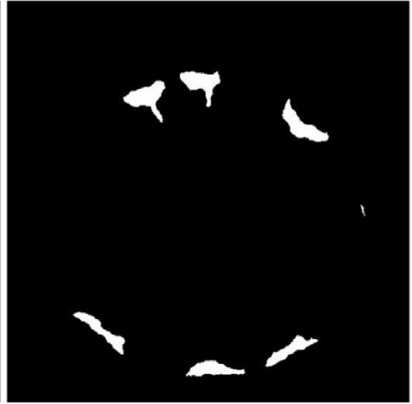

(c)

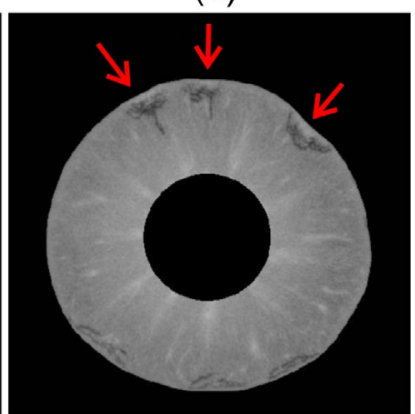

(f)

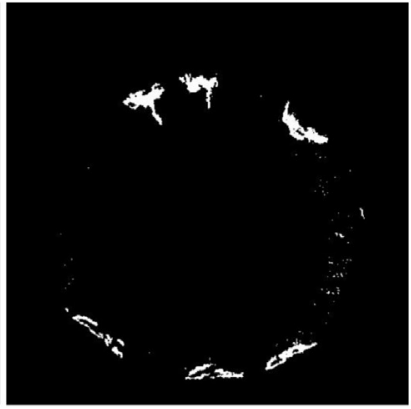

(i)

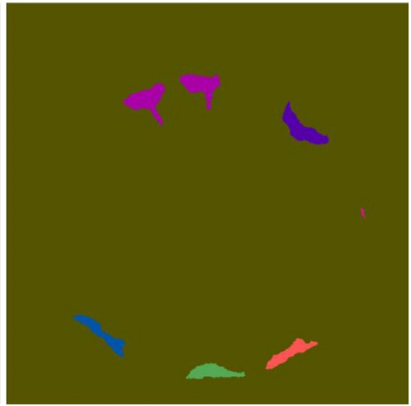

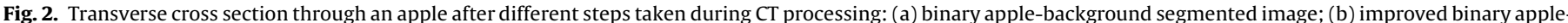

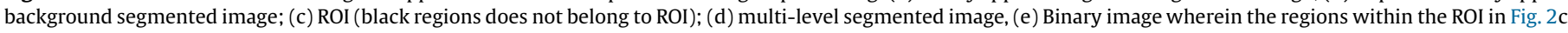

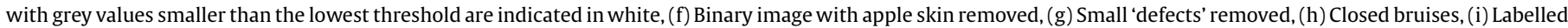
bruises (each colour corresponds to a different label). Impactor bruises are indicated by arrows in Fig. 2c.

smaller than the lowest threshold corresponded to the bruised tissue. As will be discussed later in paragraph 3.2, three discrete levels were found necessary for 'Jonagold' and 'Kanzi', whereas five discrete levels were used for 'Joly Red'. The image stacks showed that large parts of the apple skin had intensity values that also appeared in the bruised tissue (Fig. 2(e)) and that some bruises made contact with the skin. Without removing the skin from the images, the volume of the skin would be added to the volume of the bruise that made contact with it. The skin was, therefore, removed by applying an erosion operation on the segmented image in Fig. 2b using a 3D sphere with a diameter of $1.05 \mathrm{~mm}$ (5 voxels) as structuring element (Fig. 2f). Thereafter, bruises (i.e. regions in the image stacks corresponding to the discrete level with the lowest intensity values) smaller than $1.87 \mathrm{~mm}^{3}$ (200 voxels) were removed (Fig. 2g).

The bruises in the binary image stack were closed by a dilation followed by an erosion operation, using a sphere as structuring element (Fig. 2h). Next, the bruises were labelled using a 3D connected component labelling algorithm with 26-connectivity constraint (Sedgewick, 1998) (Fig. 2i). Finally, the volume of each bruise was calculated using the known voxel size and the number of voxels of each connected component. These calculated bruise volumes were manually allocated to the corresponding bruises with the help of the labelled image stack. In this way, it was guaranteed that each $V_{B}$ was allocated correctly and that no accidently caused bruise would be considered as one of the bruises caused by the pendulum. Since the apples were mounted against an anvil, the apple could become bruised at the side in contact with the anvil (called anvil bruises in this paper) as well as at the side of the impactor (called impactor bruises in this paper). In the CTimages, impactor bruises could be distinguished from anvil bruises, because the apples were always placed with the impactor bruises facing the X-ray source.

\subsection{Destructive bruise volume measurements}

The bruise volumes were measured destructively approximately one day after the CT-measurements were taken. The apple was first peeled to be able to accurately measure both bruise widths. The bruise widths, $w_{1}$ and $w_{2}$, were measured in the direction of 
the stem-calyx axis and the axis perpendicular to the stem-calyx axis, respectively. Thereafter, a section was made through the midpoint of the bruise parallel to the stem-calyx axis in order to measure the depth from the top surface of the peeled apple until the bottom of the bruise. This distance was added to the measured thickness of the skin to obtain the full bruise depth $\left(d_{b}\right)$. The depth from the apple surface to the top of the bruise $\left(d_{t}\right)$ could not be measured, because the apple was first peeled instead of cut. Therefore, it was assumed that this depth was equal to the measured thickness of the apple skin. The radius of curvature at the bruise site was measured with a digital Mitutoyo ${ }^{\mathbb{R}}$ height meter (Mitutoyo Belgium NV, Kruibeke, Belgium). The harmonic average was calculated to compensate for non-sphericity (Zarifneshat et al., 2010):

$R=\frac{2 r_{x} r_{y}}{r_{x}+r_{y}}$

where $r_{x}$ and $r_{y}$ are the radii of curvature [mm] measured in the direction of the stem-calyx axis and the axis perpendicular to the stem-calyx axis, respectively.

All formulae with an elliptical (instead of circular) bruise assumption described by Bollen et al. (1999) were investigated with special attention for the enclosed volume formula that takes the curvature at the bruise site into account. Formulae of the destructive $V_{B}$ estimation methods are as follows:

$V_{B, \text { destr }, 1}=\frac{\pi\left(d_{b}-d_{t}\right)}{24}\left(3 w_{1} w_{2}+4\left(d_{b}-d_{t}\right)^{2}\right)$,

$V_{B, \text { destr }, 2}=\frac{\pi d_{b}}{24}\left(3 w_{1} w_{2}+4 d_{b}^{2}\right)$,

$V_{B, \text { destr }, 3}=\frac{\pi}{24}\left(3 w_{1} w_{2} d_{b}+4\left(d_{b}-y\right)^{3}+4 y^{3}\right)$,

$V_{B, d e s t r, 4}=\frac{\pi}{24}\left(3 w_{1} w_{2}\left(d_{b}-d_{t}\right)+4\left(d_{b}-y\right)^{3}+4\left(d_{t}-y\right)^{3}\right)$,

$V_{B, \text { destr }, 5}=\frac{4}{3} \pi\left(\frac{\left(d_{b}-d_{t}\right) w_{1} w_{2}}{8}\right)$

where $y=R-\sqrt{R^{2}-\frac{w_{1} w_{2}}{4}}$ and $V_{B, \text { destr,3 }}$ is the destructive $V_{B}$ estimate $\left[\mathrm{mm}^{3}\right]\left(V_{B, d e s t r}\right)$ according to the enclosed volume formula.

\subsection{Statistical analysis}

To investigate to what extent the destructive $V_{B}$ estimates depend on the used $V_{B}$ estimation method, the absolute relative difference $\left(\varepsilon_{a, b}\right)$ between $V_{B, \text { destr }}$ obtained with two of the previously described $V_{B}$ formulae (Eqs. (2)-(6)) was calculated for each possible combination, using the following equation:

$\varepsilon_{a, b}(\%)=\frac{|a-b|}{a} \times 100$

in which $a$ and $b$ are $V_{B, \text { destr }}$ estimates obtained by two different destructive $V_{B}$ estimation methods.

A sensitivity analysis was performed to investigate how the $V_{B}$ estimates determined by the X-ray $\mathrm{CT}$ imaging method $\left(V_{B, C T}\right)$ change as a function of the threshold value. For each bruise, the relative change in $V_{B, C T}$ after increasing and decreasing the threshold by $5 \%$ (respectively $S_{+5 \%}$ and $S_{-5 \%}$ ) was calculated with the following formula:

$\begin{aligned} S_{\mp 5 \%} & =\frac{\left(V_{B, C T}\right)_{\text {Otsu threshold } \mp 0.05 \times \text { Otsu threshold }}-\left(V_{B, C T}\right)_{\text {Otsu threshold }}}{\left(V_{B, C T}\right)_{\text {Otsu threshold }}} \\ & \times 100 \quad\end{aligned}$

Since it becomes easier to interpret the relative changes in $V_{B, C T}$ via visualisation of the estimated bruise regions on the $C T$ images, the change in $V_{B, C T}$ was also visualised for one of the apples for relative changes in threshold between -10 and $+10 \%$ with steps of $1 \%$. Furthermore, this allowed us to check whether the $V_{B}$ estimates obtained with these different thresholds visually still seemed to correctly indicate the bruise.

To compare $V_{B, C T}$ and $V_{B \text {,destr }}$, linear regression was performed between both $V_{B}$ measures, and the absolute relative error $\left(\varepsilon_{V_{B, C T}, V_{B, \text { destr, }}}\right)$ was calculated as a percentage:

$\varepsilon_{V_{B, C T}, V_{B, \text { destr }, i}}(\%)=\frac{\left|V_{B, C T}-V_{B, \text { destr }, i}\right|}{V_{B, C T}} \times$

\section{Results and discussion}

\subsection{Destructive bruise analysis}

In Table 1 the five used pendulum release angles are summarized together with their corresponding average impact energy, maximum force, $V_{B, \text { destr,3 }}$ and bruise area $\left(\pi \frac{w_{1} w_{2}}{4}\right)$ of the impactor bruises. It can be seen that a large range of impact energies was applied, including small impact energies that resulted in small calculated bruise sizes.

The bruise area values reported in Table 1 indicate that the bruises in this work have commercially relevant dimensions, considering a total bruise surface area of $1.5 \mathrm{~cm}^{2}$ is the upper limit for apples meant for the fresh market according to the Comission of the European Union (2008). Also the peak forces in Table 1 are encountered in practice. A study by Thomson and Cotter (1993), that investigated ten apple packaging lines with an instrumented sphere, reported that the average impact size ( $>20 \mathrm{~g}$ threshold, 1 $\mathrm{g}=9.8 \mathrm{~ms}^{-2}$ ) ranged between 25 and $45 \mathrm{~g}$. Using a typical apple mass of $0.2 \mathrm{~kg}$, the applied forces given in Table 1 correspond to

Table 1

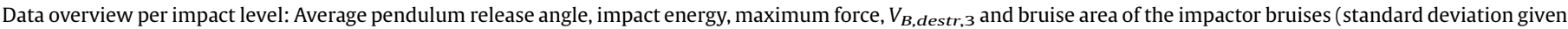
behind the \pm sign).

\begin{tabular}{|c|c|c|c|c|}
\hline Angle $\left({ }^{\circ}\right)$ & Impact energy (J) & Maximum force $(\mathrm{N})$ & $V_{B, \text { destr,3}}(\mathrm{mm} 3)$ & Bruise area $\left(\mathrm{mm}^{2}\right)$ \\
\hline $4.5 \pm 1.3$ & $0.016 \pm 0.004$ & $21.6 \pm 3.2$ & $8.2 \pm 8.2^{*}$ & $13 \pm 5^{*}$ \\
\hline $13.4 \pm 0.7$ & $0.083 \pm 0.003$ & $48.9 \pm 3.5$ & $123 \pm 30$ & $69 \pm 11$ \\
\hline $19.6 \pm 1.0$ & $0.151 \pm 0.007$ & $66.7 \pm 4.2$ & $292 \pm 83$ & $120 \pm 22$ \\
\hline $26.5 \pm 0.6$ & $0.248 \pm 0.006$ & $85.6 \pm 6.3$ & $438 \pm 123$ & $155 \pm 20$ \\
\hline $31.9 \pm 0.8$ & $0.339 \pm 0.010$ & $99.9 \pm 6.7$ & $589 \pm 172$ & $177 \pm 46$ \\
\hline
\end{tabular}

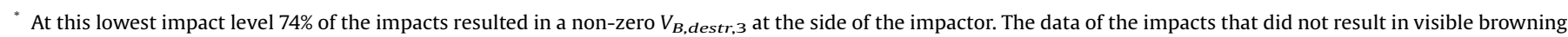
were not included in the calculation of the average $V_{B, \text { destr, } 3}$ and bruise area. 
impacts ranging from 11 to $51 \mathrm{~g}$. Note that in the study of Lin et al. (2003) much larger bruises were investigated (bruise volume of 2 to $18 \mathrm{~cm}^{3}$ ).

\subsection{CT image analysis}

The mean and standard deviations of the selected threshold values to segment the bruises were $108 \pm 4,93 \pm 13$ and $105 \pm 16$ for 'Jonagold', 'Joly Red' and 'Kanzi' apples, respectively. The algorithm was able to segment the bruises in $95.6 \%$ of the apples.

As can be seen in Figs. 2 c and 3 , bruises appear as lower intensity regions. We hypothesize that this lower X-ray absorption can be attributed to a lower water content due to drying of the bruised tissue in the time period between bruising and imaging. This corresponds to the observation of Wilson et al. (1995) that a single bruise increases the rate of moisture loss by up to $400 \%$. Hence, water released from the ruptured cells diffuses towards the apple surface where it evaporates. The dehydration of the bruised tissue can also be explained by the movement of cell content from the bruise to the surrounding tissue. Samim and Banks (1993) suggested such a dehydration effect to explain the progressive slow bruise colour fading observed after 10 to $24 \mathrm{~h}$.

Next, it was observed that in some of the CT-images of apples belonging to the three highest impact levels, a high X-ray absorption region (high intensity region) appears between the bruise (low intensity region) and the skin. Examples of this phenomenon are shown in Fig. 2c and 3c,d,e. We hypothesize that these high intensity regions are caused by compaction of pores next to the impact zone, thereby increasing tissue density (and thus X-ray absorption). Furthermore, these small pores may attract water released from broken cells deeper in the tissue through capillary action (thus further increasing X-ray absorption).

In order to test our hypotheses, one could make use of microscopy or high resolution (typically several $\mu \mathrm{m}$ ) X-ray microCT imaging of small tissue specimens. In the research of Mitsuhashi-Gonzalez et al. (2010), bruised tissue resulting from compression was studied with fluorescence microscopy and scanning electron microscopy in order to visualise dead and living cells, air spaces and cracks. Herremans et al. (2013) investigated the changes in microstructure during the development of internal browning in 'Braeburn' using high resolution X-ray micro-CT.

As mentioned in Materials and Methods, more discrete levels were used to segment the bruises in 'Joly Red' than in 'Jonagold' and 'Kanzi'. The reason for this is that in several 'Joly Red' apples, even after the erosion step for skin removal, tissue parts near the apple surface were classified as 'bruised' when only three levels were used. Using five levels for all cultivars was not possible since this could result in bad bruise segmentation in 'Jonagold' and 'Kanzi'.

This work shows that mature apple bruises can be segmented in CT-images, but in order to go towards an inline application, this method should be refined and validated more extensively in future studies. In particular, more apples should be scanned at different time intervals between bruising and scanning. It is conceivable that very young bruises will not be visible in these scans, due to insufficient fluid displacement from the bruise to its surrounding tissue. Additionally, such a study could provide valuable insight into the biology of bruise progression. Furthermore, it should be investigated whether with the recent developments in inline X-ray inspection (Janssens et al., 2016; Schryver et al., 2016), a sufficiently high acquisition and reconstruction speed is feasible for inline detection of bruises.

In many apple stacks, after completion of all imaging processing steps, tissue parts near the apple surface and mainly in the stem and calyx region were classified as bruised, whereas based on visual inspection of the CT-images one would not distinguish these regions as bruised. These are thus likely to be false-positive bruises (e.g. the bottommost "bruise" shown in Fig. 4c (in brown) and in the corresponding CT-image in Fig. 5). Since an erosion procedure using a sphere with a diameter of $1 \mathrm{~mm}$ should be sufficient to remove the epidermis and hypodermis (in the work of Verboven et al. (2008) the total thickness of these layers was observed to be less than $300 \mu \mathrm{m}$ ), this is unlikely to be the result of an unsuccessfully removed skin. A lower water content or lower tissue density in the cortex tissue parts near the apple surface is a more likely explanation. As none of them made contact with the bruises actually used in the comparison, this did not influence the results in Section 3.3. However, since bruises can be expected in

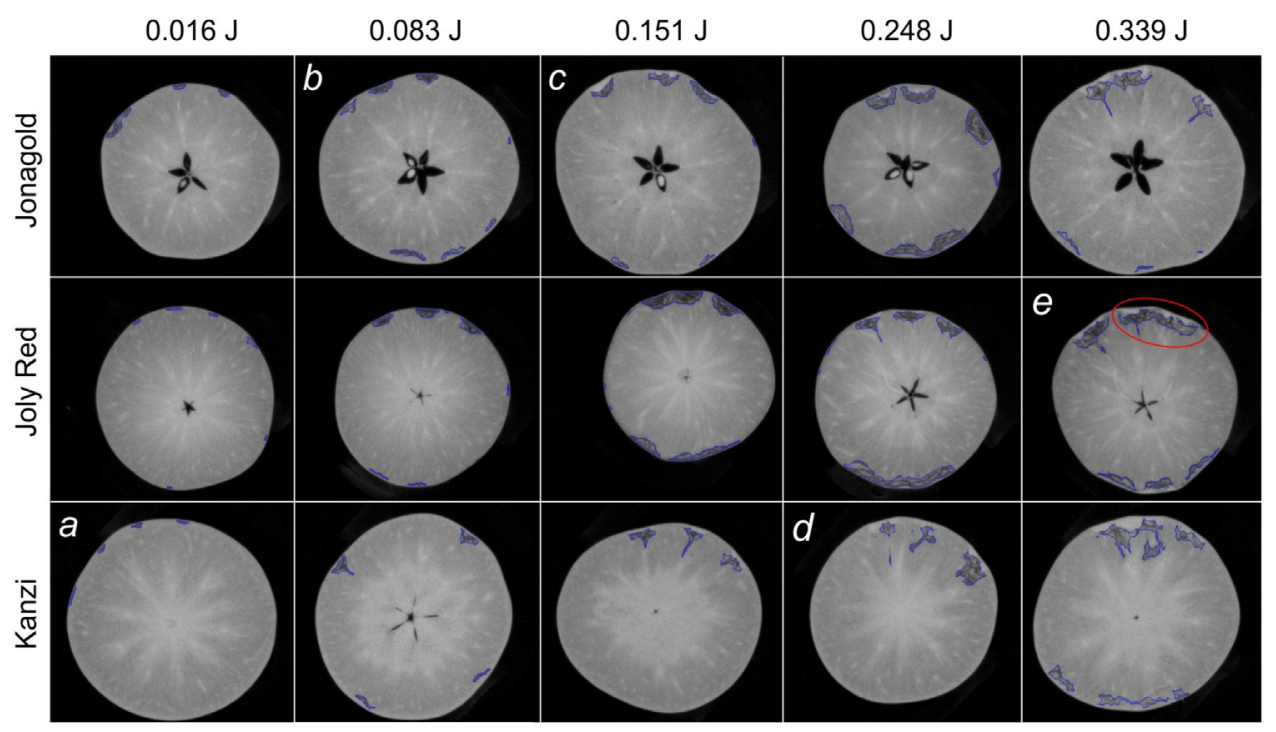

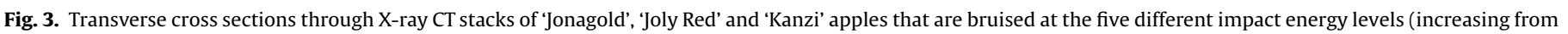

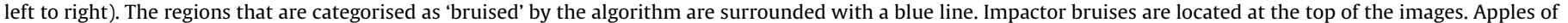

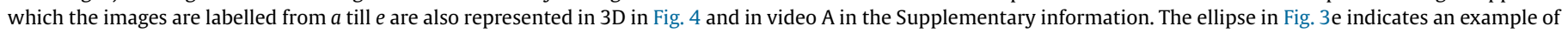
two overlapping bruises. (For interpretation of the references to colour in this figure legend, the reader is referred to the web version of this article.) 
(a)

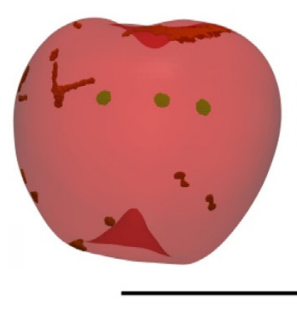

(b)

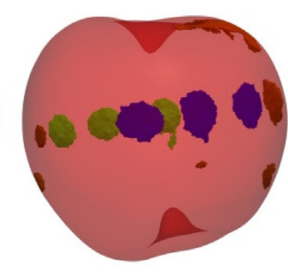

(c)

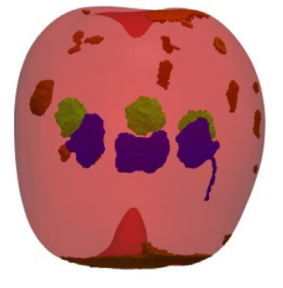

(d)

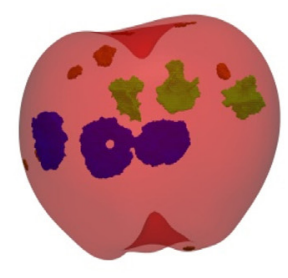

(e)

\section{Impact energy}

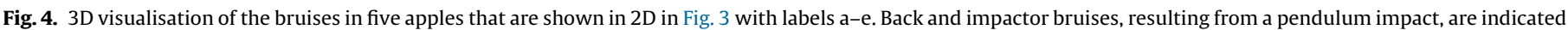

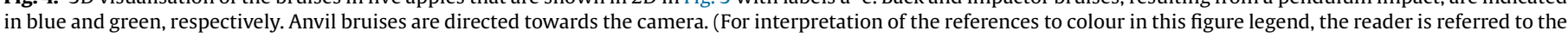
web version of this article.)

the stem and calyx region in real applications, this is a problem that should be solved to implement X-ray CT in sorting lines for bruise detection.

Fig. 3 shows that bruises can have a very irregular shape, whereas the five $V_{B}$ formulae always assume the bruise can be approximated by a an ellipsoid or a section of an ellipsoid or sphere made by a flat or convexly shaped cutting plane. This irregularity in shape typically increased for larger impact energies. In many CT images of apples bruised at the three highest impact levels, some or all impactor bruises have a thin elongated offset that protrudes in the tissue towards the core, which suggests the presence of a crack. For five of the fifteen apples shown in Fig. 3, the 3D shape of all regions that were identified as 'bruised' by the algorithm is visualized in Fig. 4. A video of these 3D reconstructed apples that turn around their stem-calyx axis can be found in the Supplementary information (Video A). The 3D presentation of the bruises confirms the previously mentioned observation of irregular bruise shapes. Lin et al. (2003), who scanned apples with MR, observed approximately cone shaped bruises. Some of the bruises in Figs. 3 and 4 can be approximated as a cone, but others are (partially)

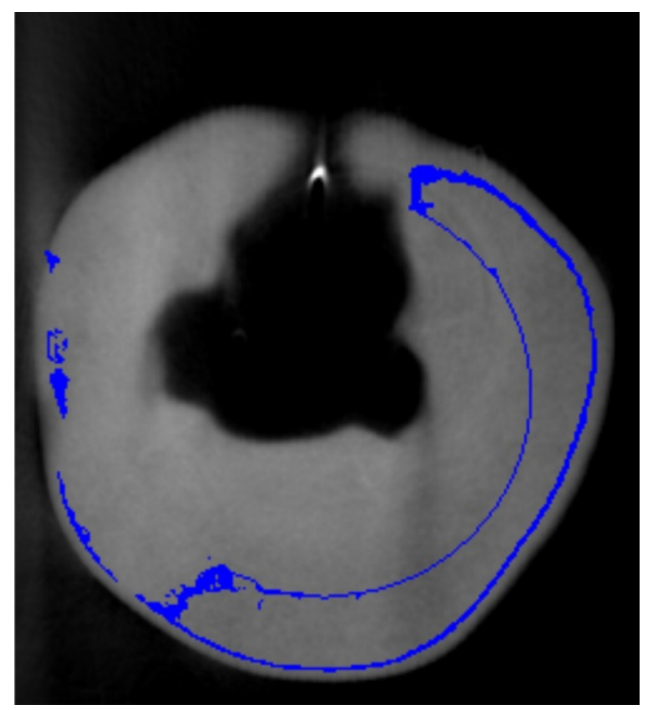

Fig. 5. CT-image taken from the stem end of the apple shown in Fig. 4 c. The regions that are categorised as 'bruised' by the algorithm are surrounded with a blue line. (For interpretation of the references to colour in this figure legend, the reader is referred to the web version of this article.) ellipsoidal, or are so irregular that no simple geometric approximation seems appropriate.

It should be noted that bruises resulting from improper handling can be shaped differently from the bruises shown Figs. 3 and 4 , since there are many factors that influence the bruise shape, such as the impactor type, impact energy and fruit maturity among others (Mowatt, 1997). The larger the impact energy, the larger the bruise volume that can be expected in case all other loading factors are the same. Fruit maturity has an influence on both bruise susceptibility and the mechanisms of bruising, but due to the optimal storage conditions in this work, we suspect that the maturity of the apples in the pendulum experiment was similar to the average maturity of apples during handling. However the material properties and/or the shape of the impactor and anvil used in this work can differ significantly from those of the many different surfaces (including other apples) an apple may collide with during handling. These discrepancies can cause differences in the stress distribution within the fruit, and therefore also differences in bruise dimensions. One can for example expect that large impacts with a flat surface will result in wider and less deep bruises than similar impacts with a spherical surface (of the same material). In this study, the apple collided with a hard spherical impactor and a hard flat anvil, while during handling the apple may for example collide with other (relatively soft) apples (typical radius of curvature: $30 \mathrm{~mm}$ ) and hard or soft flat surfaces (e.g. rubber, wood, etc.).

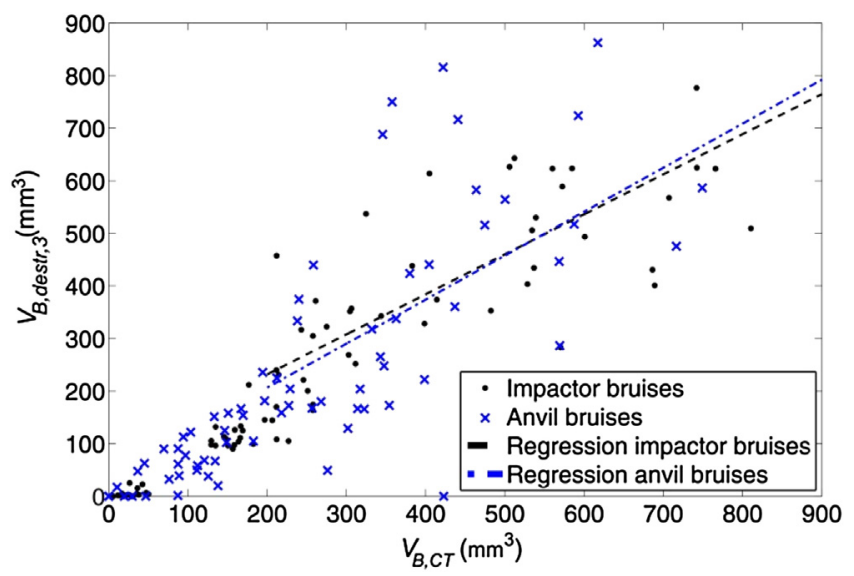

Fig. 6. Comparison of estimated $V_{B}$ using X-ray CT images with $V_{B}$ estimates determined by destructive measurements using the enclosed volume formula. 
Table 2

Coefficient of determination $\left(R^{2}\right)$, intercept and slope of the linear regression fits between $V_{B, d e s t r, i}$ (for $i=1,2, \ldots, 5$ ) and $V_{B, C T}$, and the averages and corresponding

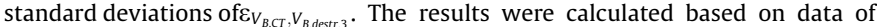
45 impactor bruises with $V_{B, C T}>200 \mathrm{~mm}^{3}$.

\begin{tabular}{llllll}
\hline & $V_{B, \text { destr }, 1}$ & $V_{B, \text { destr }, 2}$ & $V_{B, \text { destr,3 }}$ & $V_{B, \text { destr }, 4}$ & $V_{B, \text { destr,5 }}$ \\
\hline $\mathrm{R}^{2}$ & 0.57 & 0.59 & 0.59 & 0.57 & 0.59 \\
Intercept & 57.06 & 78.06 & 79.46 & 66.03 & 64.12 \\
Slope & 0.72 & 0.82 & 0.76 & 0.69 & 0.84 \\
$\quad \varepsilon_{V_{B, C T}, V_{B, \text { destr }, i}}(\%)$ & $27 \pm 21$ & $26 \pm 23$ & $25 \pm 21$ & $27 \pm 20$ & $27 \pm 21$
\end{tabular}

A last observation is that there is no abrupt transition in the original CT-images from the 'bruised tissue' to the 'healthy tissue' (see Fig. 2c). As will be discussed in Section 3.4, varying the threshold led to highly variable $V_{B, C T}$ calculations.

\subsection{Comparison of destructive and CT-based bruise volume estimation method}

Several bruises were removed for further analysis since they overlapped with their neighbouring bruise (e.g. the two overlapping impactor bruises in Fig. 3e). 83 impactor bruises and 78 anvil bruises remained.

The volumes of the impactor and anvil bruises based on destructive measurements and CT scans are compared with each other in Fig. 6 and Tables 2 and 3. Fig. 6 indicates that the data are heteroscedastic, thus fitting a regression line over the entire range of bruise volumes would lead to an unreliable coefficient of determination. Regression analysis and calculation of the absolute relative error was only performed on the data with $V_{B, C T}>200$ $\mathrm{mm}_{3}$. As can be derived from Table 2 and 3, the coefficient of determination between $V_{B, \text { destr,i}}$ and $V_{B, C T}$ was more than two times larger for impactor bruises than for anvil bruises. This lower correlation for anvil bruises can be attributed to difficulties that were encountered during the destructive measurements. Anvil bruises were often less brown than impactor bruises, making it difficult to visually distinguish the damaged tissue from the healthy tissue. ${ }^{1}$ The fact that these anvil bruises could be distinguished more easily in the CT-images, suggest that CT imaging is a more reliable technique than destructive measurements to estimate $V_{B}$. The comparatively less browning at the backside indicates a lower concentration of damaged cells, which can be attributed to energy dissipation through cell distortion and collapse of air spaces rather than through cell damage. Moreover, the anvil bruises often had a larger diameter and a smaller depth than the impactor bruises, as a result of the flat surface of the anvil against which the back of the apple impacted, resulting in larger contact areas and lower stresses than for the hemispherical impactor.

The results of the comparison between $V_{B, \text { destr }}$ and $V_{B, C T}$ of the impactor bruises in Table 2 does not show any large differences between the five $V_{B}$ formulae. The slope is always smaller than 1 , ranging between 0.69 and 0.84 , and the intercept is always larger than zero, ranging between 57.06 and 79.46 . The coefficients of determination ranged between 0.57 and 0.59 . The absolute relative error in volume with $V_{B, d e s t r}$ as compared with $V_{B, C T}$ is lowest in case the enclose volume formula $\left(V_{B, d e s t r, 3}\right)$ was used $(25 \pm 21 \%)$.

\footnotetext{
${ }^{1}$ In several cases (mainly in anvil bruises) the ellipsoidal shaped bruise area looked rather whitish instead of brownish. This white tissue was also considered "bruised".
}

Table 3

Coefficient of determination $\left(\mathrm{R}^{2}\right)$, intercept and slope of the linear regression fits between $V_{B, \text { destr,i }}$ (for $i=1,2, \ldots, 5$ ) and $V_{B, C T}$, and the averages and corresponding standard deviations of $\varepsilon_{V_{B C T}} . V_{B \text { destr } 3}$. The results were calculated based on data of 39 anvil bruises with $V_{B, C T}>200 \mathrm{~mm}^{3}$.

\begin{tabular}{llllll}
\hline & $V_{B, \text { destr, } 1}$ & $V_{B, \text { destr }, 2}$ & $V_{B, \text { destr, } 3}$ & $V_{B, \text { destr, } 4}$ & $V_{B, \text { destr, },}$ \\
\hline $\mathrm{R}^{2}$ & 0.27 & 0.27 & 0.27 & 0.27 & 0.27 \\
Intercept & 3.58 & 34.78 & 39.09 & 10.54 & 15.84 \\
Slope & 0.80 & 0.89 & 0.84 & 0.77 & 0.96 \\
$\quad \varepsilon_{V_{B, C}, V_{B, \text { dest }, i}}(\%)$ & $42 \pm 27$ & $41 \pm 31$ & $40 \pm 29$ & $41 \pm 26$ & $45 \pm 34$ \\
\hline
\end{tabular}

However, the absolute difference with the average relative errors of the other four $V_{B}$ formulae is maximally $2 \%$.

No further data-analysis seemed appropriate for the smaller bruises (especially for bruises with $V_{B, C T}<100 \mathrm{~mm}^{3}$ ), because in this data region the CT-based method is measuring a large range of $V_{B}$ while the destructive method is not. This can be explained by the poor resolution of the conventional destructive method. Note that Fig. 6 displays several data points (five anvil bruises and six impactor bruises) with zero $V_{B, \text { destr,3 }}$ and non-zero $V_{B, C T}$. It may be that there actually was discoloured tissue in these cases, but that it was peeled off since (with exception of one such bruise ${ }^{2}$ ) the corresponding X-ray CT images revealed small bruises very close to the skin. Another explanation is that the X-ray CT images may have revealed damaged tissue that was not discoloured.

Since, with the present data, we cannot prove whether the threshold-based algorithm only classifies the brown tissue as 'bruised', it is possible that the CT-based bruise volumes are an under- or overestimation of the discoloured tissue volumes. When we assume the 'correct' $V_{B}$ is equal to the discoloured tissue region (i.e. what is observed by the consumer and what is tried to be quantified in conventional bruise volume measurements), overestimation of $V_{B}$ could be due to the fact that the tissue in the outer regions of $V_{B, C T}$ might not be brown, but might have different $\mathrm{X}$-ray absorption properties than the unaffected tissue due to biochemical and/or physiological changes occurring in these tissue regions. We hypothesize that such biochemical and/or physiological changes in non-brown tissue regions can be explained by a very low percentage of ruptured cells in these tissue regions or by diffusion of cell content (enzymes, water, etc.) from ruptured cells, located in the neighbouring brown tissue, towards these nonbrown tissue regions. Note that although we call it 'an overestimation' (since we compare to the brown tissue volume), it should be seen as an advantage of CT instead of a source of error since this indicates that CT can visualise tissue changes (resulting from bruises) that are not visible by the eye but still may cause quality loss. To verify how well the Otsu threshold in this X-ray CTbased $V_{B}$ measurement method manages to distinguish between non-brown and brown tissue, the X-ray CT measurements should be repeated on apples of which the bruises are afterwards analysed via RGB images of thin parallel sections through the bruises.

'Incorrect' $V_{B}$ estimates by the X-ray CT-based method might also be due to an improper thresholding, since the contrast between healthy and bruised tissue is low in the X-ray CT images. To test whether a better contrast can be obtained with MR, several

\footnotetext{
2 The other two anvil bruises in the apple that contained the anvil bruise with zero $V_{B, \text { destr }}$ and $V_{B, C T}=423 \mathrm{~mm}^{3}$ showed only slightly discoloured tissue (they were rather whitish) and were difficult to distinguish from the healthy tissue. Thus in this particular bruise (i.e., the bruise with zero $V_{B, \text { destr }}$ and $V_{B, C T}=423 \mathrm{~mm}^{3}$ ), the X-ray CT image probably revealed damaged tissue that was not discoloured.
} 
apples should be scanned with both X-ray CT and MR. MR often performs better in the visualisation of water-related effects, but it also has its limitations. The most important limitations of MR are the limited resolution in comparison to $\mathrm{CT}$ and the cumbersome selection process of the optimal MR sequence for detection in the application (Herremans et al., 2014a; Lammertyn et al., 2003; Winisdorffer et al., 2015). Note that the low contrast transition in CT images can be the result of gradual transitions happening within the tissue, such as a gradual change in the percentage of damaged cells, tissue browning, porosity and/or water content. For a deeper investigation in future research of what is occurring in these 'bruised'-to-'healthy'-transition-regions, we suggest to combine X-ray CT imaging with RGB (visualising browning) and/ or microscopy imaging (visualising living and damaged cells) of sections through the bruises.

\subsection{Sensitivity analysis}

When the threshold was increased by $5 \%$, more bruises overlapped or made contact with possible false-positive bruises near the skin. This can be explained by the fact that in some apples, the larger threshold caused a larger region near the skin to be classified as bruised. Moreover, in case the threshold was decreased by $5 \%$, some bruises could not be detected anymore. Therefore, from the 161 bruises ( 83 impactor and 78 anvil bruises), 29 and 13 bruises could not be used in the calculation of, respectively, the average $S_{+5 \%}$ and $S_{-5 \%} . V_{B, C T}$ enlarged by $26 \pm 16 \%$ and declined by $19 \pm 8 \%$ when the threshold was respectively increased or decreased by $5 \%$. A $S_{+5 \%}$ of $26 \%$ corresponds to an increase in bruise widths and depth by only $8 \%$ when the bruise shape is equal to a section of sphere (e.g., an extra border of thickness $0.4 \mathrm{~mm}$ when considering a bruise of width $10 \mathrm{~mm}$ and depth $5 \mathrm{~mm}$ ). Thus, although $S_{+5 \%}$ and $S_{-5 \%}$ are large, the thickness of the border added to or subtracted from $V_{B, C T}$ using the Otsu threshold is rather small and acceptable considering the gradual changes in water content, browning intensity, etc. that can be expected in the border of a bruise (see Section 3.3 for related discussion). Visualisation of the boundaries of $V_{B, C T}$ corresponding to different thresholds (see Video 1 ) showed that thresholds that lie between $-10 \%$ and $+5 \%$ from the Otsu threshold seemed visually to still be able to correctly separate the bruised tissue from the healthy tissue. Above a change in threshold of $+5 \%$, some or all bruises in the apple shown in Video 1 made contact with each other.

In addition, just like $V_{B, C T}$ largely depends on the threshold, $V_{B}$, destr largely depends on the chosen formula. Table 4 shows that the relative difference between $V_{B, \text { destr }}$ obtained with different destructive $V_{B}$ estimation methods can be large. Furthermore, the destructive measures are subject to biases. For example, the measured bruise depth is not necessarily equal to the maximum bruise depth, since the measured $d_{b}$ will depend on the exact location of the cut made through the bruise. Hence, despite the large threshold sensitivity, which can be a limitation for the applicability of the technique, we expect that X-ray CT-based $V_{B}$ estimates are not less accurate than estimates made by destructive bruise measurements (Table 3 ).

\section{Table 4}

Average $\varepsilon_{a, b}$ as defined in Eq. (7) and its corresponding standard deviation. The results were calculated based on the data of all 215 bruises with non-zero $d_{b}-d_{t}, w_{1}$ and $w_{2}$.

\begin{tabular}{lccccc}
\hline$b$ & $V_{B, \text { destr, } 1}$ & $V_{B, \text { destr,2 }}$ & $V_{B, \text { destr,3 }}$ & $V_{B, \text { destr,4 }}$ & $V_{B, \text { destr,5 }}$ \\
$a$ & & & & & \\
\hline$V_{B, \text { destr, } 1}$ & $/$ & $27 \pm 18$ & $22 \pm 19$ & $3 \pm 5$ & $21 \pm 7$ \\
$V_{B, \text { destr, }}$ & $20 \pm 9$ & $/$ & $4 \pm 2$ & $20 \pm 7$ & $7 \pm 8$ \\
$V_{B, \text { destr,3 }}$ & $16 \pm 11$ & $4 \pm 2$ & $/$ & $16 \pm 8$ & $8 \pm 8$ \\
$V_{B, \text { destr,4 }}$ & $3 \pm 4$ & $25 \pm 13$ & $20 \pm 14$ & $/$ & $21 \pm 7$ \\
$V_{B, \text { destr,5 }}$ & $17 \pm 5$ & $8 \pm 12$ & $9 \pm 11$ & $17 \pm 5$ & $/$ \\
\hline
\end{tabular}

\section{Conclusion}

X-ray CT measurements of artificially bruised apples from different cultivars were successfully used to quantify bruises in apple cultivars for a large range of impact levels. Two and three dimensional visualisation of the bruises resulted in the observation that bruises, resulting from an impact by a pendulum with a spherical impactor, can expose a very irregular shape, especially at high impact energies. Such irregular bruise shapes imply that the $V_{B}$ estimates based on simple geometric assumptions will not deliver accurate results. A sensitivity analysis indicated that the size of the X-ray CT-based $V_{B}$ estimates is very sensitive to changes in the threshold value.

\section{Acknowledgements}

We thank the European Union's Seventh Framework Program for research technological development and demonstration through the PicknPack (311987) project and the Agency for Innovation by Science and Technology in Flanders (IWT) through the Chameleon (SB-100021) and TomFood (SB-120033) projects. This research was carried out in the context of the European COST Action FA1106 ('QualiFruit'). At last, we thank Greet Kerckhofs for her advice for using the Multi-level version of Otsu's method.

\section{Appendix A. Supplementary data}

Supplementary data associated with this article can be found, in the online version, at http://dx.doi.org/10.1016/j. postharvbio.2017.01.013.

\section{References}

Baritelle, A.L., Hyde, G.M., 2001. Commodity conditioning to reduce impact bruising Postharvest Biol. Technol. 21 (3), 331-339. doi:http://dx.doi.org/10.1016/S09255214(00)00154-X.

Bollen, A.F., Nguyen, H.X., Dela Rue, B.T., 1999. Comparison of methods for estimating the bruise volume of apples. J. Agric. Eng. Res. 74 (4), 325-330. doi: http://dx.doi.org/10.1006/jaer.1999.0468.

Cantre, D., Herremans, E., Verboven, P., Ampofo-Asiama, J., Nicolaï, B., 2014. Characterization of the 3-D microstructure of mango (Mangifera indica L. cv. Carabao) during ripening using X-ray computed microtomography. Innov. Food Sci. Emerg. Technol. 24, 28-39. doi:http://dx.doi.org/10.1016/j.ifset.2013.12.008.

Chen, P., Yazdani, R., 1991. Prediction of apple bruising due to impact on different surfaces. Am. Soc. Agric. Eng. 34 (3), 956-961.

Comission of the European Union, 2008. Commission regulation (EC) No 1221/2008. Off. J. Eur. Union L 336, 1-80.

Diels, E., Odenthal, T., Keresztes, J., Vanmaercke, S., Verboven, P., Nicolaï, B., et al., 2016. Development of a visco-elastoplastic contact force model and its parameter determination for apples. Postharvest Biol. Technol. 120, 157-166. doi:http://dx.doi.org/10.1016/j.postharvbio.2016.06.003.

Diener, R.G., Mitchell, J.P., Rhoten, M.L., 1970. Using an X-ray image scan to sort bruised apples. Agric. Eng. 51 (6), 356-361.

Donis-González, I.R., Guyer, D.E., Pease, A., Barthel, F., 2014. Internal characterisation of fresh agricultural products using traditional and ultrafast electron beam Xray computed tomography imaging. Biosyst. Eng. 117 (1),104-113. doi:http://dx. doi.org/10.1016/j.biosystemseng.2013.07.002.

Herremans, E., Verboven, P., Bongaers, E., Estrade, P., Verlinden, B.E., Wevers, M., et al., 2013. Characterisation of Braeburn browning disorder by means of X-ray micro-CT. Postharvest Biol. Technol. 75, 114-124. doi:http://dx.doi.org/10.1016/ j.postharvbio.2012.08.008.

Herremans, E., Melado-Herreros, A., Defraeye, T., Verlinden, B., Hertog, M. Verboven, P., et al., 2014a. Comparison of X-ray CT and MRI of watercore disorder of different apple cultivars. Postharvest Biol. Technol. 87, 42-50. doi: http://dx.doi.org/10.1016/j.postharvbio.2013.08.008.

Herremans, E. Verboven, P., Defraeye, T., Rogge, S., Ho, QT., Hertog, M.L.A.T.M., et al. 2014b. X-ray CT for quantitative food microstructure engineering: the apple case. Nucl. Inst. Methods Phys. Res. Sect. B 324, 88-94. doi:http://dx.doi.org/ 10.1016/j.nimb.2013.07.035.

Janssens, E., Pereira, L.F.A., Beenhouwer, J., De, Dael, Van, M., Verboven, P., Nicolaï, B. Sijbers, J., 2016. Fast Inline Inspection of Apples by a Neural Network Based Filtered Backprojection Method (iCT). , pp. 2-7.

Kotwaliwale, N., Singh, K., Kalne, A., Jha, S.N., Seth, N., Kar, A., 2014. X-ray imaging methods for internal quality evaluation of agricultural produce. J. Food Sci. Technol. 51 (1), 1-15. doi:http://dx.doi.org/10.1007/s13197-011-0485-y. 
Lammertyn, J., Dresselaers, T., Van Hecke, P., Jancsók, P., Wevers, M., Nicolaï, B.M. 2003. MRI and X-ray CT study of spatial distribution of core breakdown in conference pears. Magn. Reson. Imaging 21, 805-815. doi:http://dx.doi.org/ 10.1016/S0730-725X(03)00105-X

Li, Z., Thomas, C., 2014. Quantitative evaluation of mechanical damage to fresh fruits. Trends Food Sci. Technol. 35 (2), 138-150. doi:http://dx.doi.org/10.1016/j. tifs.2013.12.001.

Lin, T.T., Cheng, Y.C., Yu, J.F., 2003. Assessment of internal bruise volume of selected fruits using MR imaging. ASAE Annual Meeting Paper 1-16.

Lu, L.X., Wang, Z.W., 2007. Dropping bruise fragility and bruise boundary of apple fruitTrans. ASAE 50 (4), 1323-1329. . Retrieved from http://asae.frymulti.com/ abstract.asp?adid $=23609 \& \mathrm{t}=3$.

Magwaza, L.S., Opara, U.L., 2014. Investigating non-destructive quantification and characterization of pomegranate fruit internal structure using X-ray computed tomography. Postharvest Biol. Technol. 95, 1-6. doi:http://dx.doi.org/10.1016/j. postharvbio.2014.03.014.

Mitsuhashi-Gonzalez, K., Pitts, M.J., Fellman, J.K., Curry, E.A., Clary, C.D., 2010. Bruising profile of fresh apples associated with tissue type and structure. Appl. Eng. Agric. 26 (3), 509-518.

Mowatt, C.M., 1997. Factors Influencing Tthe Susceptibility of Apples to Brusing. Massey University.

Otsu, N., 1979. A threshold selection method from gray-level histograms. IEEE Trans. Syst. Man Cybern. 9 (1), 62-66. doi:http://dx.doi.org/10.1109/ TSMC.1979.4310076.

Pang, W., Studman, C.J., Ward, G.T., 1992. Bruising damage in apple-to-apple. J. Agric. Eng. Res. 52, 229-240.

Pang, D.W., Studman, C.J., Banks, N.H., 1994. Apple bruising thresholds for an instrumented sphere. Am. Soc. Agric. Eng. 37 (3), 893-897.

Samim, W., Banks, N.H., 1993. Color changes in apple bruises over time. Acta Hortic 304-306.
Schatzki, T.F., Haff, R.P., Young, R., Can, I., Le, L.-C., Toyofuku, N., 1997. Defect detection in apples by means of X-ray imaging. Trans. ASAE 40 (5), 1407-1415.

Schryver, T., De Dhaene, J., Dierick, M., Boone, M.N., Janssens, E., Sijbers, J., et al. 2016. NDT \& E international in-line NDT with X-Ray CT combining sample rotation and translation. NDT E Int. 84, 89-98. doi:http://dx.doi.org/10.1016/j. ndteint.2016.09.001.

Sedgewick, R., 1998. Algorithms in C, 3rd ed Addison-Wesley ISBN:0-201-31452-5.

Thomson, G., Cotter, D., 1993. Assessment of post harvest bruising of export apples with instrumented sphere technology. Hortic. Res. Dev. Corp. 57.

Tollner, E.W., Hung, Y.-C., Upchurch, B.L., Prussia, S.E., 1992. Relating X-ray absorption to density and water content with apples. Trans. ASAE 35 (6), 1921 1928.

VCBT, 2014. Jaarverslag 2013. Leuven.

Van Zeebroeck, M., 2005. The Discrete Element Method (DEM) to Simulate Fruit Impact Damage During Transport and Handling. KU Leuven.

Verboven, P., Kerckhofs, G., Mebatsion, H.K., Ho, Q.T., Temst, K., Wevers, M., et al., 2008. Three-dimensional gas exchange pathways in pome fruit characterized by synchrotron X-Ray computed tomography. Plant Physiol. 147 (2), 518-527. doi: http://dx.doi.org/10.1104/pp.108.118935.

Wilson, L.G., Boyette, M.D., Estes, E.A., 1995. Postharvest Handling and Cooling O Fresh Fruits, Vegetables and Flowers for Small Farms. Horticulure Information Leaflet 802-804. North Carolina cooperative extension service.

Winisdorffer, G., Musse, M., Quellec, S., Devaux, M.F., Lahaye, M., Mariette, F., 2015 MRI investigation of subcellular water compartmentalization and gas distribution in apples. Magn. Reson. Imaging 33 (5), 671-680. doi:http://dx.doi. org/10.1016/j.mri.2015.02.014.

Zarifneshat, S., Ghassemzadeh, H.R., Sadeghi, M., Abbaspour-Fard, M.H., Hamid, E. Javadi, A., Shervani-Tabar, M.T., 2010. Effect of impact level and fruit properties on golden delicious apple bruising. Am. J. Agric. Biol. Sci. 5 (2), 114-121. doi: http://dx.doi.org/10.3844/ajabssp.2010.114.121. 